Research Article

\title{
Tenacissoside $H$ Induces Apoptosis and Inhibits Migration of Colon Cancer Cells by Downregulating Expression of GOLPH3 Gene
}

\author{
Zhong-Shi Hong $\left(\mathbb{D}\right.$, Hai-Bin Zhuang $\mathbb{D}^{\circ}$, Cheng-Zhi Qiu $\mathbb{D}^{D}$, Ze-Sheng Shi $\mathbb{D}$, \\ Chun-Xiao Wang $\mathbb{D}$, Zhi-chuan Chen $\mathbb{D}$, and Jian-peng Pan \\ Department of General Surgery, The Second Affiliated Hospital of Fujian Medical University, Quanzhou 362000, \\ Fujian Province, China \\ Correspondence should be addressed to Cheng-Zhi Qiu; qchengzhi@fjmu.edu.cn
}

Received 24 December 2019; Revised 7 April 2020; Accepted 17 April 2020; Published 7 May 2020

Academic Editor: Fatima Martel

Copyright (c) 2020 Zhong-Shi Hong et al. This is an open access article distributed under the Creative Commons Attribution License, which permits unrestricted use, distribution, and reproduction in any medium, provided the original work is properly cited.

\begin{abstract}
Objective. Tenacissoside $\mathrm{H}(\mathrm{TDH})$ is a Chinese medicine monomer extracted from Marsdenia tenacissima extract (MTE), which has been confirmed to have antitumor effects, but its mechanism is still unclear. The aim of this study was to investigate the effect and mechanism of TDH on human colon cancer LoVo cell proliferation and migration and explore the correlation of TDH treatment with the expression of GOLPH3 and cell signaling pathways in LoVo cells. Methods. LoVo cells were treated with TDH at $0.1,1,10$, and $100 \mu \mathrm{g} / \mathrm{mL}$ for 24,48 , and $72 \mathrm{~h}$. The proliferation rate of LoVo cells was evaluated by MTT assay. Recombinant plasmid p-CMV-2-GOLPH3 was constructed, and p-CMV-2-GOLPH3 and p-CMV-2 empty plasmids were transfected into LoVo cells by lipofection. Western blotting was used to detect the transfection efficiency and the expression of p-p70S6K, p70S6K, $\beta$-catenin, and GOLPH3. The apoptosis rate was analyzed with Annexin V-FITC/PI double-staining method, and cell migration assessed by transwell assay. Results. TDH inhibited the proliferation of LoVo cells in a concentration-dependent manner. The IC50 of TDH treatment in LoVo cells at 24,48 , and $72 \mathrm{~h}$ was $40.24,13.00$, and $5.73 \mu \mathrm{g} / \mathrm{mL}$, respectively. TDH treatment significantly induced apoptosis and suppressed the viability and migration of human colon cancer LoVo cells. The effect of TDH on induction of apoptosis and inhibition of migration in LoVo cells decreased significantly after activating the PI3K/AKT/mTOR and Wnt/ $\beta$-catenin signaling pathways with agonists. Additionally, the expression of GOLPH3 protein downregulated significantly in LoVo cells under TDH treatment. Overexpression of the GOLPH3 gene increased the expression of key proteins in PI3K/AKT/mTOR and $\mathrm{Wnt} / \beta$-catenin signaling pathways and blocked the antitumor activity of TDH. Conclusion. Collectively, the present results indicated that TDH can inhibit the proliferation vitality of colon cancer LoVo cells through downregulating GOLPH3 expression and activity of $\mathrm{PI} 3 \mathrm{~K} / \mathrm{AKT} / \mathrm{mTOR}$ and $\mathrm{Wnt} / \beta$-catenin signaling pathways.
\end{abstract}

\section{Introduction}

Colon cancer is one of the most common malignant tumors of the digestive tract. The incidence and mortality rate keep increasing worldwide [1]. Surgical resection is the most important means of treatment at present. However, patients in the advanced stage often received chemotherapy and targeted therapy according to individual conditions. In China, traditional Chinese medicine also plays an important role in the adjuvant treatment of malignant tumors. Studies have shown that many Chinese herbal extracts can inhibit tumor growth and improve quality of life [2]. Through chemical composition analysis and pharmacological studies, it was found that the extract contains different effective chemical ingredients, and the molecular mechanism of its anticancer effect is necessary for further research.

Marsdenia tenacissima is the dry vine, root, or leaf of the genus $M$. tenacissima (Roxb.) Wight et Arn, and the isolated M. tenacissima extract (MTE) causes obvious inhibitory effect on various malignant cells [3]. Tenacissoside is a kind 
of major active ingredient in MTE; Tenacissoside $\mathrm{H}(\mathrm{TDH})$ is one of its monomers, molecular formula: $\mathrm{C}_{42} \mathrm{H}_{66} \mathrm{O}_{14}$, belonging to the $\mathrm{C} 21$ steroidal glycosides and is considered to be an antitumor active substance isolated from $M$. tenacissima [4]. However, the inhibitory effect and molecular mechanism of TDH on colon cancer is still unfamiliar.

GOLPH3 has been validated as an oncoprotein and its expression in colon cancer tissues was significantly increased compared to normal tissues [5]; GOLPH3 overexpression can upregulate activation of the $\mathrm{PI} 3 \mathrm{~K} / \mathrm{AKT} / \mathrm{mTOR}$ and $\mathrm{Wnt} / \beta$-catenin signaling pathways, promote proliferation, and induce apoptosis in colon cancer cells. Meanwhile, silencing expression of the GOLPH3 gene can reverse the resistance of HT29 colon cancer cells to 5-fluorouracil and cisplatin [6-8].

It is unclear whether the antitumor effects of $\mathrm{TDH}$ are related to downregulation of GOLPH3 gene expression and cellular signaling pathway. In the present study, we investigated the effect and mechanism of TDH on the proliferation, apoptosis, and migration in human colon cancer LoVo cells.

\section{Materials and Methods}

2.1. Drugs and Reagents. The Human colon cancer cell line LoVo (Shanghai Institute of Cell Science, Chinese Academy of Sciences); TDH (20 mg/branch; batch number: 111913201803; China Food and Drug Control Institute); RPMI1640 medium (Beijing Suolaibao Bioscience and Technology Co., Ltd.); pFLAG-CMV-2 vector (Sigma, St. Louis, MO, USA); cDNA reverse transcription kit (Invitrogen, Carlsbad, CA, USA); Lipofectamine $\mathrm{TM}^{\mathrm{TM}} 3000$ transfection reagent (Invitrogen); Opti-MEM serum-free medium (Shanghai Tuoran Biotechnology Co., Ltd.); rabbit anti-human GOLPH3 polyclonal antibody (ab98023; Abcam, Cambridge, UK); phosphorylated ribosomal protein S6 kinase $\beta 1$ (p-p70S6K) antibody (sc-377529; Santa Cruz Biotechnology, Santa Cruz, USA); p70S6K antibody (sc-8418; Santa Cruz Biotechnology); $\beta$-catenin antibody (sc-7199; Santa Cruz Biotechnology); horseradish peroxidase-labeled goat anti-rabbit IgG antibody (A0208; Beyotime, Nanking, China); WB and IP protein lysate (Shanghai Lai Maple Biotechnology Co., Ltd.); trypsin powder, 3-(4,5-dimethylthiazol-2-yl)-2,5-diphenyltetrazolium bromide (MTT), dimethyl sulfoxide (DMSO), propidium iodide (PI) staining solution, and fetal bovine serum (FBS) (Sigma); Annexin-V detection kit (Becton Dickinson, Franklin Lakes, NJ, USA); BCA Protein Quantification Kit (Shanghai Weijin Biotechnology Co., Ltd.); Luminol (Shanghai Pu Zhen Biological Technology Co., Ltd.); and tetramethylethylenediamine (TEMED; Beijing BioDee BioTech Corp. Ltd.).

2.2. Cell Lines and Culture. The human colon cancer cell line LoVo was purchased from Shanghai Institute of Cell Science, Chinese Academy of Sciences. The LoVo cell line was cultured in RPMI $1640+10 \%$ FBS and placed in an incubator at $37^{\circ} \mathrm{C}$ in $5 \% \quad \mathrm{CO}_{2}+95 \%$ air in a saturated humidity environment.
2.3. Construction of GOLPH3 Overexpression Plasmid and Cell Transfection. Construction and identification of recombinant plasmid: total RNA in LoVo cells was extracted and reverse transcribed to obtain $\mathrm{CDNA}$, which was subjected to polymerase chain reaction (PCR) amplification (underlined as an enzyme cleavage site) using the following primers: forward, TTTAAGCTTATGACCTCGCTGACCCAGC; reverse, TTTTCTAGATTACTTGGTGAACGCCGCC. The PCR amplification product was a full-length cDNA of 897 bp GOLPH3 full-length cDNA (NM_022130). The PCR product was digested with HindIII and $\mathrm{XbaI}$ and ligated into the pFlagCMV-2 vector after double digestion. The clones were selected and verified by sequencing to obtain the GOLPH3 overexpression vector.

Lipofectamine transfection: (1) cells were digested in the exponential phase 1 day before transfection and plated on six-well plates at $105 /$ well; cells were suitable for transfection when they reached $80 \%$ confluence; (2) lipofectamine 3000 liposomes were diluted in Opti-MEM and mixed well; (3) empty vector plasmid and recombinant plasmid were diluted with Opti-MEM medium; the premix was prepared and then added with the $\mathrm{P} 3000^{\mathrm{TM}}$ reagent in the kit and mixed well; (4) lipofectamine 3000 liposome was added in a ratio of $1: 1$ with empty vector plasmid and recombinant plasmid premix and was incubated for $5 \mathrm{~min}$ at room temperature; and (5) empty plasmid-liposome complexes and recombinant plasmid-liposome complexes were added to the cell culture, and experimental tests were performed.

\subsection{MTT Assay to Evaluate the Proliferation of LoVo Cells.} After blood cell counting, LoVo cells $\left(2 \times 10^{3}\right)$ were inoculated onto 96-well plates at $100 \mu \mathrm{L} /$ well. After $24 \mathrm{~h}$, different concentrations of TDH were added. At 24, 48, and $72 \mathrm{~h}$ after drug treatment, $10 \mu \mathrm{L} 5 \mathrm{mg} / \mathrm{mL}$ MTT solution was added to each well and reacted at $37^{\circ} \mathrm{C}$ for $4 \mathrm{~h}$. Each well added with DMSO and OD490 was determined by using a microplate reader. IC50 was calculated using the Logit method via comparing the OD490 values at different time points.

2.5. Cell Grouping. The cells were divided into the following groups: (1) Control, LoVo cells; (2) TDH:TDH-treated LoVo cells; (3) Experimental Group 1, LoVo cells treated with TDH and PI3K/AKT/mTOR signaling pathway agonist insulin-like growth factor (IGF)-1; (4) Experimental Group 2 , LoVo cells treated with TDH and $\mathrm{Wnt} / \beta$-catenin signaling pathway agonist Wnt agonist 1 ; (5) p-CMV-2:LoVo cells transfected with p-CMV-2 empty plasmid; (6) GOLPH3: LoVo cells transfected with p-CMV-2-GOLPH3 recombinant plasmid; (7) Experimental Group 3, TDH-treated LoVo cells transfected with empty plasmid; (8) Experimental Group 4: TDH-treated LoVo cells overexpressing GOLPH3 recombinant plasmid. Recombinant plasmid p-CMV-2GOLPH3 was constructed, and p-CMV-2-GOLPH3 and p-CMV-2 empty plasmids were transfected into human colon cancer LoVo cells by lipofection. 
2.6. Annexin V-FITC/PI Double-Staining Method to Detect Apoptosis Rate. LoVo cells were digested and seeded in sixwell plates $\left(5 \times 10^{4}\right.$ cells $)$. After $24 \mathrm{~h}$, cells were treated with different concentrations of drug according to the experimental group. The treated cells were aspirated in a suitable centrifuge tube, washed once with phosphate-buffered saline (PBS), and the appropriate amount of trypsin cell digest was added. Trypsin-EDTA solution was removed when the adherent cells were obtained. We avoided excessive trypsin digestion. The cell culture solution collected in the abovementioned step was added, mixed, transferred to a centrifuge tube, and centrifuged at $1000 \times g$ for $5 \mathrm{~min}$. The supernatant was discarded and the cells were collected and gently resuspended in PBS and counted. Resuspended cells $(50,000-100,000)$ were centrifuged at $1,000 \times g$ for $5 \mathrm{~min}$, the supernatant was discarded, and cells were gently resuspended by adding $195 \mu \mathrm{L}$ Annexin V-fluorescein isothiocyanate (FITC) binding solution. We added $5 \mu \mathrm{L}$ Annexin V-FITC and mixed gently. We then added $10 \mu \mathrm{L}$ of propidium iodide (PI) stain and mixed gently. The cells were incubated at room temperature $\left(20^{\circ} \mathrm{C}-25^{\circ} \mathrm{C}\right)$ for $10-20 \mathrm{~min}$ in the dark and placed in an ice bath. Aluminum foil was used to protect the cells from light. The cells were resuspended two or three times during the incubation to improve the staining effect. Flow cytometry was performed immediately, Annexin V-FITC showed green fluorescence, and PI was red fluorescence.

2.7. Transwell Assay for Cell Migration. The LoVo cells were cultured to logarithmic growth and digested, washed, and suspended in serum-free medium, and the concentration was adjusted to $2 \times 10^{5}$ cells; in the lower chamber (i.e., the bottom of the 24 -well plate), $600-800 \mu \mathrm{L}$ culture medium containing $10 \%$ serum was added, $100 \mu \mathrm{L}$ of cell suspension was added to the upper chamber, and different drug treatments were performed according to the experimental group. After continuing culture for $24 \mathrm{~h}$, the chamber was carefully removed with forceps; the upper chamber liquid was blotted and transferred to the well and added with $800 \mu \mathrm{L}$ methanol, fixed at room temperature for $30 \mathrm{~min}$, rinsed with PBS, and soaked three times. The chamber was then taken out, the upper chamber liquid was removed, and the cells on the surface membrane of the upper chamber bottom with a wet cotton swab were transferred to the lower chamber containing $500 \mu \mathrm{L}$ of crystal violet and stained at room temperature for $30 \mathrm{~min}$; The cells were observed under an inverted microscope, photographed, and counted.

2.8. Western Blotting for Protein Expression. Cells in the logarithmic growth phase were collected and washed once with PBS, lysed in RIPA lysis buffer, and kept on ice for $20 \mathrm{~min}$. The protein concentration was determined by the BCA method, and the total protein of cells extracted by cell lysis was used for SDS-PAGE. After electrophoresis, proteins were transferred to nitrocellulose membranes. PBST containing 5\% skimmed milk powder was sealed at room temperature for $60 \mathrm{~min}$. The blots were incubated with rabbit anti-human GOLPH3 polyclonal antibody ( $1: 1000)$,
p-p70S6K antibody $(1: 1000)$, p70S6K antibody $(1$ : $1000)$, and $\beta$-catenin antibody $(1: 1000)$ at $4^{\circ} \mathrm{C}$ overnight and rinsed three times with PBST for $20 \mathrm{~min}$. The corresponding horseradish peroxidase-labeled secondary antibodies were added and incubated for $1 \mathrm{~h}$ at room temperature and rinsed three times with PBST for $10 \mathrm{~min}$ and detected with ECL chemiluminescence reagent. The image was subjected to gray-scale analysis using Image-J version 1.48 (National Institutes of Health, Bethesda, MD, USA), and the relative intensity of target protein expression is equal to the ratio of the gray value of the target protein band to the gray value of the GADPH band.

2.9. Statistical Analysis. Statistical analysis was performed using SPSS version 20.0. All samples were tested to ascertain if they followed the homogeneity of variance. The paired $t$ test was used for comparison between two groups and oneway analysis of variance was used for comparison between $\geq 3$ groups. The least significant difference method was used for multiple comparisons between groups, and $P<0.05$ indicated that the difference was statistically significant.

\section{Results}

3.1. TDH Inhibits the Proliferation of LoVo Cells. MTT assay was used to detect the LoVo cell proliferation under TDH treatment at concentrations of $0.1,1,10$, and $100 \mu \mathrm{g} / \mathrm{ml}$ for 24,48 , and $72 \mathrm{~h}$, and the respective growth inhibition curves were drawn (Figure 1). The results showed $\mathrm{TDH}$ inhibited significantly LoVo cell proliferation in a concentrationdependent manner (Table 1). However, under the same $\mathrm{TDH}$ concentration, there was no significant difference in the inhibitory effect of TDH on LoVo cell proliferation as the treatment time was prolonged $(P>0.05)$. It suggested that there was no obvious time-dependent effect of TDH. The IC50 of TDH treatment in LoVo cells at 24, 48, and $72 \mathrm{~h}$ was $40.24,13.00$, and $5.73 \mu \mathrm{g} / \mathrm{ml}$, respectively. In the subsequent experiments, the concentration of TDH was selected to be $25 \mu \mathrm{g} / \mathrm{ml}$.

3.2. TDH Induces Apoptosis of LoVo Cells. Apoptosis was detected by Annexin V-FITC/PI flow cytometry. The apoptosis rates of the Control Group, the TDH Group, Experimental Group 1, and Experimental Group 2 were $0.51 \pm 0.54 \%, 31.77 \pm 3.47 \%, 1.47 \pm 0.97 \%$, and $2.68 \pm 1.79 \%$, respectively. The apoptosis rate of the TDH Group was significantly higher than the Control Group $(P<0.01)$. The apoptosis rate of Experimental Group 1 and Experimental Group 2 was significantly lower than the TDH Group $(P<0.05)$ (Figure 2). This result indicated that the PI3K/ $\mathrm{AKT} / \mathrm{mTOR}$ or $\mathrm{Wnt} / \beta$-catenin signaling pathway agonist could attenuate the apoptosis induced by TDH in LoVo cells.

3.3. TDH Inhibits LoVo Cell Migration. The numbers of cell migration in the Control Group, the TDH Group, Experimental Group 1, and Experimental Group 2 were $293 \pm 64$, $47 \pm 12,277 \pm 23$, and $253 \pm 35$, respectively (Figure 3 ). The 


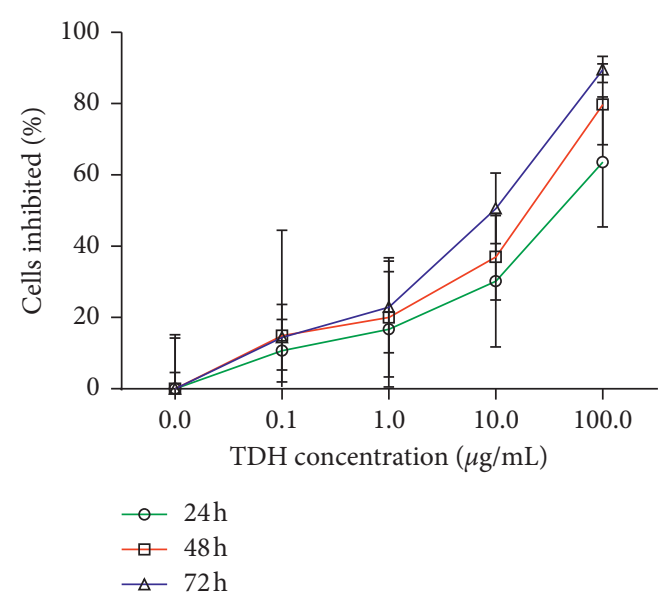

FIGURE 1: Inhibition of LoVo cells proliferation under different concentrations of TDH treatment. The IC50 of TDH in LoVo cells at 24,48 , and $72 \mathrm{~h}$ was $40.24,13.00$, and $5.73 \mu \mathrm{g} / \mathrm{ml}$.

TABLE 1: Proliferation inhibition rates of LoVo cells under TDH treatment.

\begin{tabular}{lccc}
\hline $\begin{array}{l}\text { TDH } \\
\text { concentration } \\
(\mu \mathrm{g} / \mathrm{mL})\end{array}$ & \multicolumn{3}{c}{ Proliferation inhibition rate $(\%)$} \\
\hline 0 & $24 \mathrm{~h}$ & $48 \mathrm{~h}$ & $72 \mathrm{~h}$ \\
0.1 & $0.00 \pm 15.06$ & $0.00 \pm 4.53$ & $0.00 \pm 14.22$ \\
& $10.72 \pm 8.78 \mathrm{NS}$ & $14.98 \pm 29.49$ & $14.42 \pm 9.19 \mathrm{NS}$ \\
1 & $16.74 \pm 16.20$ & $\mathrm{NS}$ \\
10 & $\mathrm{NS}$ & $\mathrm{NS}$ & $22.96 \pm 12.80^{* *}$ \\
100 & $30.17 \pm 18.43^{*}$ & $37.00 \pm 12.22^{* *}$ & $50.62 \pm 9.88^{* * *}$ \\
\hline
\end{tabular}

Results are shown as mean \pm SD. Comparisons of different concentrations in the same time (NS $P>0.05,{ }^{*} P<0.05,{ }^{* *} P<0.01,{ }^{* * *} P<0.001$ ). $24 \mathrm{~h}$, $F=9.725 ; 48 \mathrm{~h}, F=12.959 ; 72 \mathrm{~h}, F=44.726$.

migrated cells in the TDH Group were significantly decreased than the Control Group $(P<0.05)$. Meanwhile, compared with the TDH Group, the migrated cells in Experimental Group 1 and Experimental Group 2 increased significantly $(P<0.05)$. The experiments showed that signal pathway agonists could reduce the inhibitory effect of TDH on migration in LoVo cells.

3.4. TDH Affects LoVo Cell Signaling Pathway-Related Protein Expression. Compared with the Control Group, expression of $\mathrm{p}$-p70S6K and $\beta$-catenin in the TDH Group was significantly decreased $(P<0.05)$, and the corresponding signaling pathway was inhibited (Table 2, Figure 4 ). After adding the signaling pathway agonist (IGF-1 or Wnt agonist 1), expression of p-p70S6K in Experimental Group 1 and $\beta$-catenin expression in Experimental Group 2 were significantly higher than in the TDH Group $(P<0.05)$. Moreover, under TDH treatment, the GOLPH3 expression in LoVo cells was also altered, and the expression of GOLPH3 protein in the TDH Group was significantly lower than in the Control Group $(P<0.01)$. These results demonstrated that TDH could downregulate both GOLPH3 expression and activation of the PI3K/AKT/mTOR and $\mathrm{Wnt} / \beta$-catenin signaling pathway in LoVo cells.

3.5. Effect of TDH on the Expression of Signaling PathwayRelated Proteins in LoVo Cells Overexpressed GOLPH3. Compared with the p-CMV-2 Group, expression of GOLPH3 protein in the GOLPH3 Group increased significantly $(P<0.05)$; it indicated that the transfection efficiency was good (Table 3, Figure 5). Moreover, expression of p-p70S6K, $\beta$-catenin, and GOLPH3 proteins in Experimental Group 4 was increased significantly than Experimental Group $3 \quad(P<0.05)$. It implied that GOLPH3 overexpression can hinder the inhibitory effect of TDH on PI3K/AKT/mTOR and $\mathrm{Wnt} / \beta$-catenin signaling pathways in LoVo cells.

3.6. Effect of TDH on Apoptosis in LoVo Cells Overexpressed GOLPH3. Apoptosis was detected by Annexin V-FITC/PI flow cytometry. Apoptosis rates in the p-CMV-2 Group, the GOLPH3 Group, Experimental Group 3, and Experimental Group 4 were $1.11 \pm 0.65 \%, 0.34 \pm 0.35 \%, 30.06 \pm 4.86 \%$, and $2.27 \pm 1.19 \%$ (Figure 6). The apoptosis rate of Experimental Group 3 was significantly higher than the p-CMV-2 Group, while the apoptosis rate of Experimental Group 4 was significantly lower than Experimental Group $3(P<0.01)$. This result testified that GOLPH3 overexpression in LoVo cells could arrest the TDH-induced apoptosis.

3.7. Effect of TDH on Migration in LoVo Cells Overexpressed GOLPH3. The numbers of cell migration in the p-CMV-2 Group, the GOLPH3 Group, Experimental Group 3, and Experimental Group 4 were $301 \pm 26,372 \pm 55,39 \pm 14$, and $284 \pm 31$, respectively(Figure 7 ). The number of migrated cells in Experiment Group 3 was significantly decreased than the p-CMV-2 Group $(P<0.01)$. Correspondingly, the number of migrated cells in Experimental Group 4 was also significantly higher than that in Experimental Group 3 $(P<0.01)$. The experiments manifested that the inhibitory effect of TDH on migration in LoVo cells overexpressed GOLPH3 significantly weakened.

\section{Discussion}

In China, the incidence and mortality of colorectal cancer continue to rise [9]. Although chemotherapy and targeted drugs can improve the survival rate, they are expensive and have obvious adverse effects. To improve the life quality of patients with advanced colon cancer by comprehensive treatment, traditional Chinese medicine is used to assist patients with colon cancer. It can effectively reduce the adverse effects of chemotherapy, improve immunity, and play an important role in the treatment of colon cancer [10]. $M$. tenacissima is widely used as a traditional Chinese medicine in clinical practice; its preparation Xiaoaiping is used for adjuvant treatment in various malignant tumors; it was interesting to note that the therapeutic effect is remarkable and the adverse reactions are few [11-13]. The 

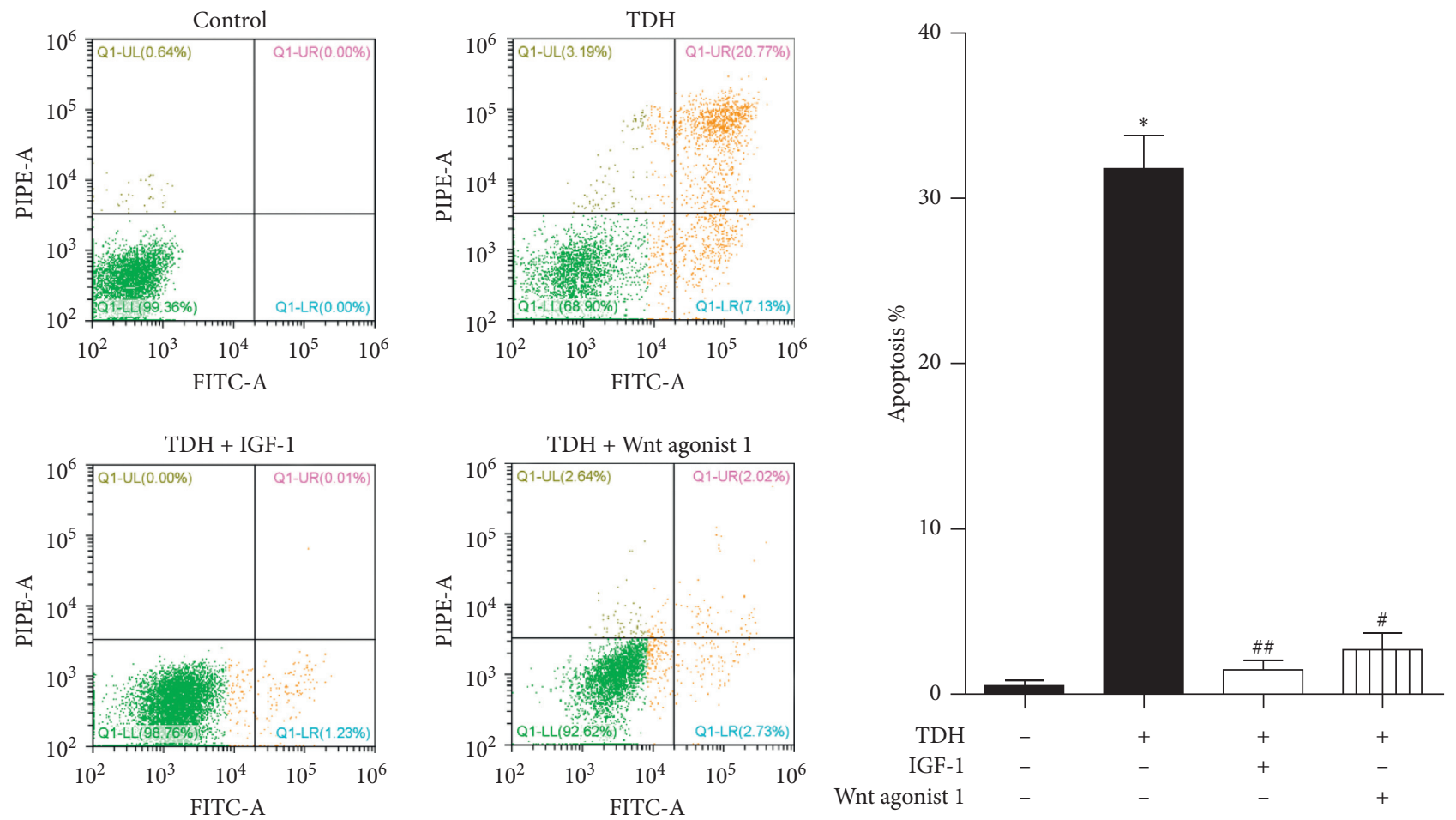

Figure 2: Apoptosis rate of LoVo cells under TDH treatment. Comparison of the TDH Group with the Control Group $\left({ }^{* *} P<0.01\right)$. Comparisons of Experimental Group 1 and Experimental Group 2 with the TDH Group $\left({ }^{\#} P<0.01,{ }^{\#} P<0.01\right)$.

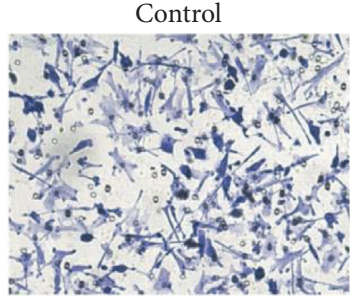

$\mathrm{TDH}+\mathrm{IGF}-1$

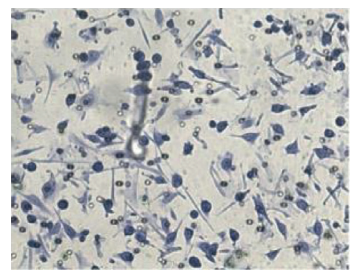

$\mathrm{TDH}$

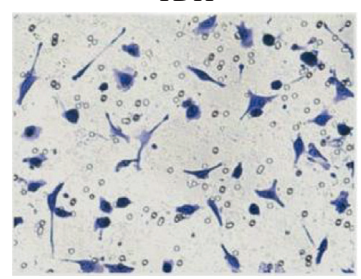

$\mathrm{TDH}+$ Wnt agonist 1

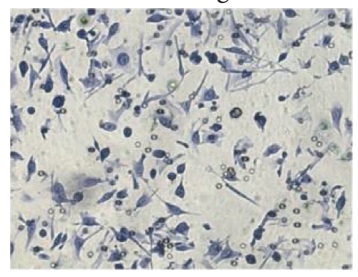

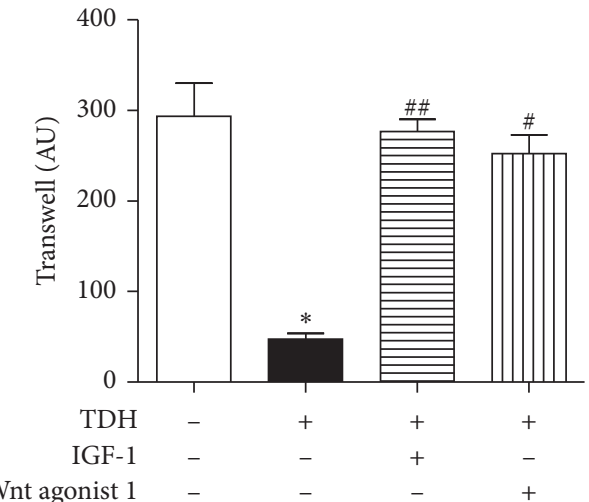

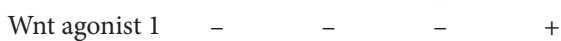

Figure 3: LoVo cell migration under TDH treatment. Comparison of the TDH Group with the Control Group $\left({ }^{*} P<0.05\right)$. Comparisons of Experimental Group 1 and Experimental Group 2 with the TDH Group $\left({ }^{\#} P<0.05,{ }^{\# \#} P<0.01\right)$.

TABLE 2: Expression levels of proteins under TDH treatment.

\begin{tabular}{lcc}
\hline Group & p-p70S6K/p70S6K & $\beta$-Catenin \\
\hline Control & $1.00 \pm 0.10$ & $1.00 \pm 0.06$ \\
TDH & $0.30 \pm 0.17^{*}$ & $0.12 \pm 0.06^{* * *}$ \\
TDH + IGF-1 & $0.83 \pm 0.18^{\#}$ & $0.80 \pm 0.22^{\#}$ \\
TDH + Wnt agonist 1 & $0.71 \pm 0.15 \mathrm{NS}$ & $0.70 \pm 0.17^{\#}$ \\
\hline
\end{tabular}

Results are shown as mean \pm SD. The TDH Group compared with the Control Group $\left({ }^{*} P<0.05,{ }^{* * *} P<0.001\right)$; Experimental Group 1 and Experimental Group 2 compared with the TDH Group (NS $P>0.05$, $\left.{ }^{\#} P<0.05\right)$. composition of $M$. tenacissima is complex, and its antitumor mechanism is still unclear and needs further study.

Related studies have shown that TDH can inhibit the proliferation of esophageal cancer cells by regulating the cell cycle and reducing proliferating cell nuclear antigen expression [14]. TDH can also inhibit the growth of mouse lung cancer cells, reduce the number of lung metastases, and enhance the immune function of tumor-bearing mice by regulating Th1/Th2-related cytokines [15]. We found that TDH had a significant concentration-dependent inhibitory 


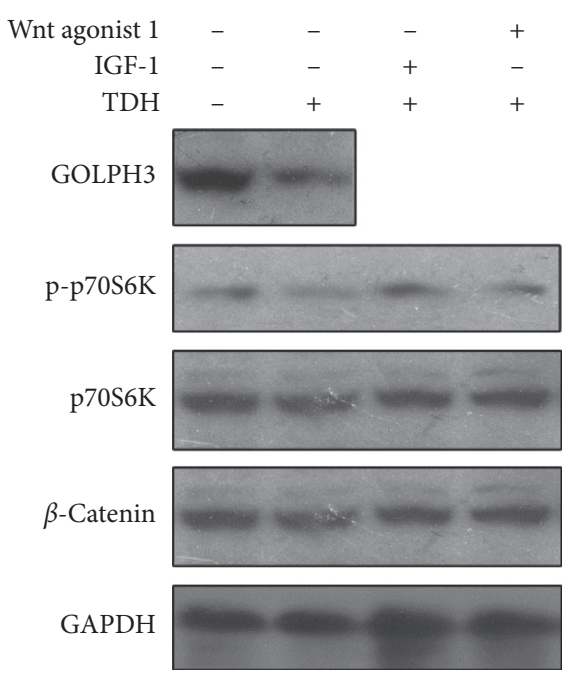

(a)

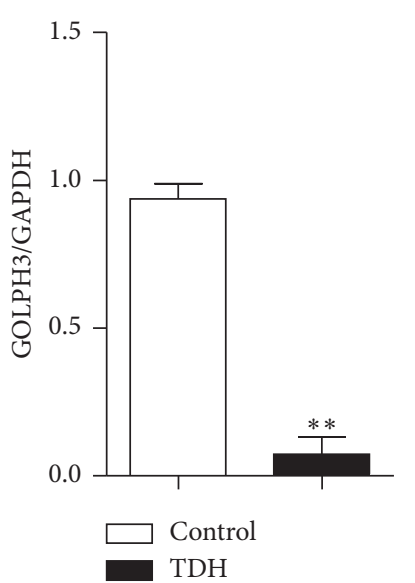

(b)

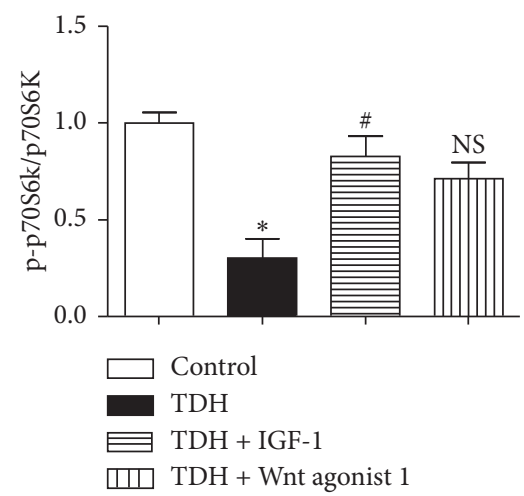

(c)

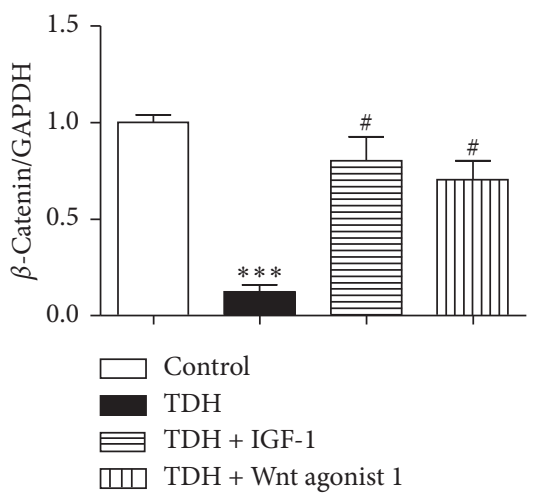

(d)

FIGURE 4: (a) Expression of related proteins in LoVo cells under TDH treatment. (b) The TDH Group compared with the Control Group $\left({ }^{*} P<0.05,{ }^{* *} P<0.01,{ }^{* * *} P<0.001\right)$; (c) Experimental Group 1 and (d) Experimental Group 2 compared with the TDH Group (NS $P<0.05$, $\left.{ }^{\#} P<0.05\right)$.

TABLE 3: Expression levels of proteins in LoVo cells overexpressed GOLPH3 under TDH treatment.

\begin{tabular}{lccc}
\hline Group & $\begin{array}{c}\text { p-p70S6K/ } \\
\text { p70S6K }\end{array}$ & $\beta$-Catenin & GOLPH3 \\
\hline pCMV-control & $1.00 \pm 0.18$ & $1.00 \pm 0.11$ & $1.00 \pm 0.27$ \\
pCMV-GOLPH3 & $1.29 \pm 0.18^{*}$ & $1.63 \pm 0.06^{* *}$ & $3.82 \pm 0.52^{*}$ \\
TDH + pCMV- & $0.29 \pm 0.05^{*}$ & $0.24 \pm 0.07^{*}$ & $0.17 \pm 0.12^{*}$ \\
control & $1.24 \pm 0.17^{\# \#}$ & $1.82 \pm 0.44^{\#}$ & $3.57 \pm 0.55^{\#}$ \\
TDH + pCMV- & & & \\
GOLPH3 & &
\end{tabular}

Results are shown as mean \pm SD. The GOLPH3 Group and Experimental Group 3 compared with the p-CMV-2 Group $\left({ }^{*} P<0.05,{ }^{* *} P<0.01\right)$; Experimental Group 4 compared with Experimental Group $3\left({ }^{\#} P<0.05\right.$, $\#$ \# $P<0.01)$.

effect on the proliferation of human colon cancer LoVo cells in vitro; moreover, it can also induce apoptosis and inhibit the migration of LoVo cells; these results indicated that TDH could inhibit colon cancer cell viability and metastasis. The abnormal activation of the PI3K/AKT/mTOR signaling pathway has been observed in a variety of human tumors, including esophageal, ovarian, breast, and colorectal cancer and promoted the development of tumor by promoting cell proliferation, inhibiting apoptosis, or activating angiogenesis [16-19]. The aberrant activation of the Wnt/ $\beta$-catenin signaling pathway has been also demonstrated in colorectal cancer and regulates gene expression and cell invasion, migration, proliferation, and differentiation in the initiation and progression of CRC [20]. In the present study, under $\mathrm{TDH}$ treatment, the expression of $\mathrm{p}$-p70S6K and $\beta$-catenin was significantly reduced in LoVo cells; however, the expression of $\mathrm{p}$-p70S6K and $\beta$-catenin was upregulated, apoptosis decreased, and cell migration increased after using mTOR and Wnt signaling pathway agonists; the result suggested that $\mathrm{TDH}$ may exert antitumor activity through inhibiting the PI3K/AKT/mTOR and Wnt/ $\beta$-catenin signaling pathways in the colon cancer.

In addition, we also found that the decrease of GOLPH3 expression was detected in LoVo cells under TDH treatment. GOLPH3 is localized in the Golgi apparatus, widely involved in many important physiological processes, and plays an important role in glycosyltransferases location, glycosylation, and secretory trafficking which were related to cancer development. GOLPH3 is a oncogene that is frequently overexpressed in various 


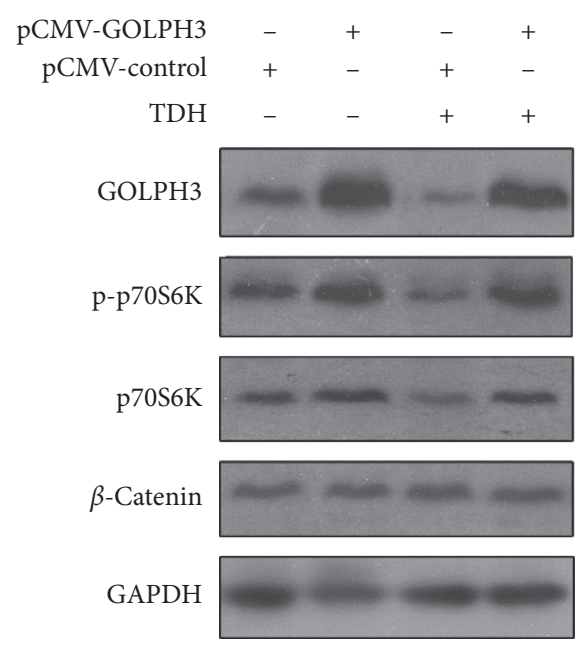

(a)

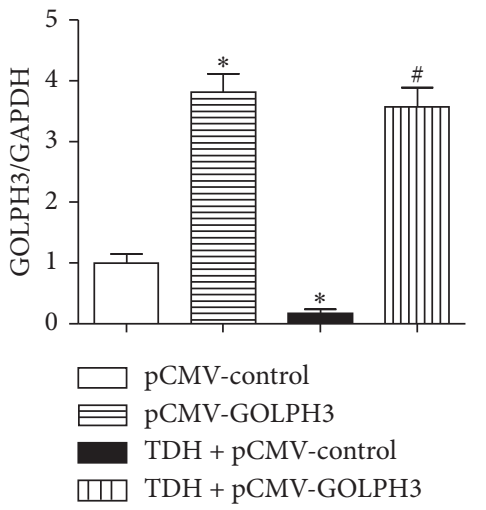

(b)

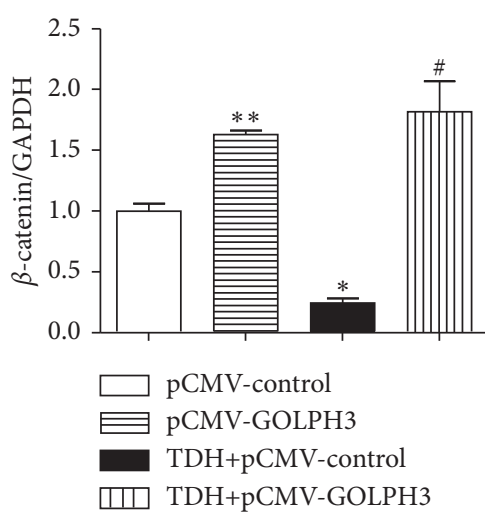

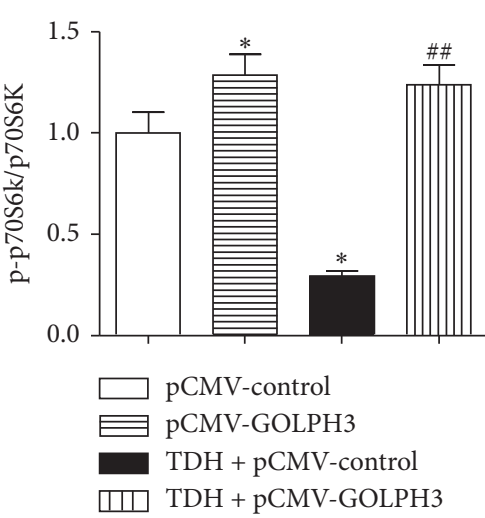

(c)

(d)

FIGURE 5: (a) Expression of related proteins in LoVo cells overexpressed GOLPH3 under TDH treatment. (b) The GOLPH3 Group and (c) Experimental Group 3 compared with the p-CMV-2 Group $\left({ }^{*} P<0.05,{ }^{* *} P<0.01\right)$; (d) Experimental Group 4 compared with Experimental Group $3\left({ }^{\#} P<0.05,{ }^{\#} P<0.01\right)$.
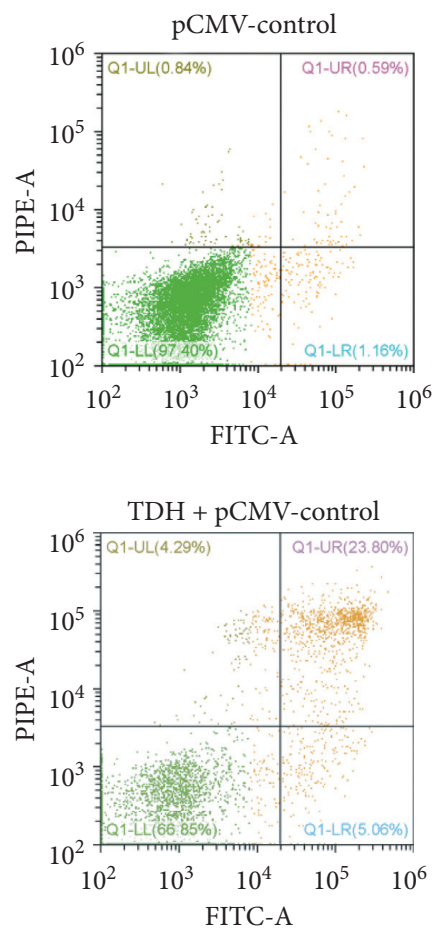
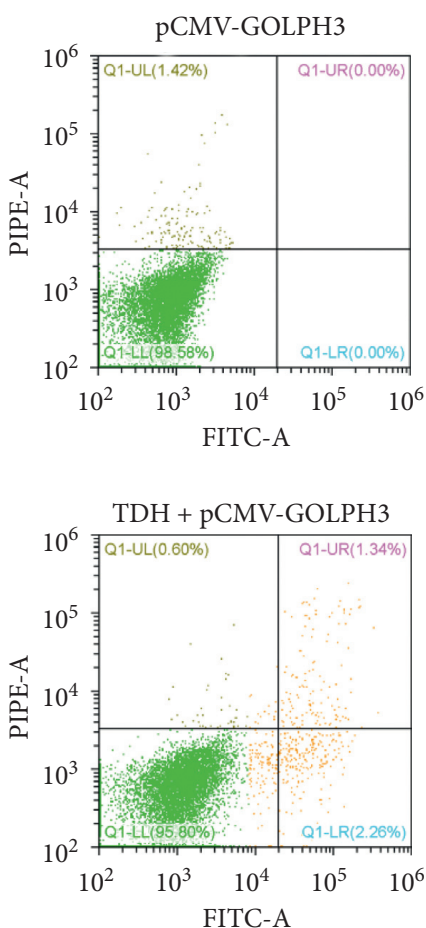

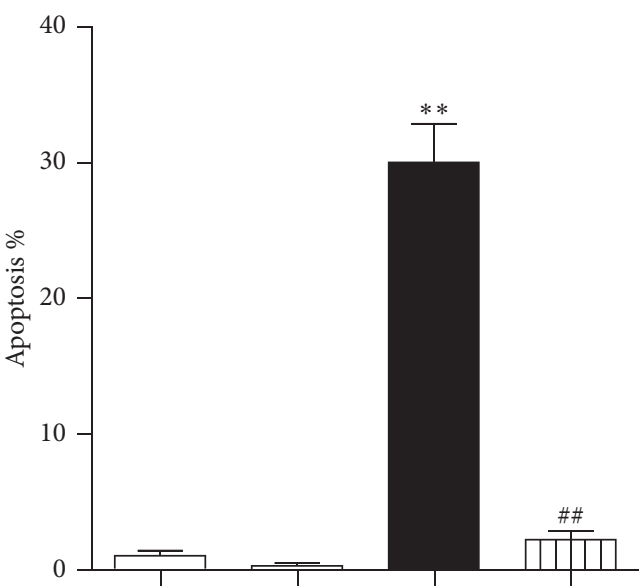

$\begin{array}{rrrrr}\text { TDH } & - & - & + & + \\ \text { pCMV-GOLPH3 } & - & + & - & + \\ \text { pCMV-control } & + & - & + & -\end{array}$

Figure 6: Apoptosis rate of LoVo cells overexpressed GOLPH3 under TDH treatment. Experimental Group 3 compared with the p-CMV-2 Group $\left({ }^{* *} P<0.01\right)$; Experimental Group 4 compared with Experimental Group $3\left({ }^{\# \#} P<0.01\right)$. 

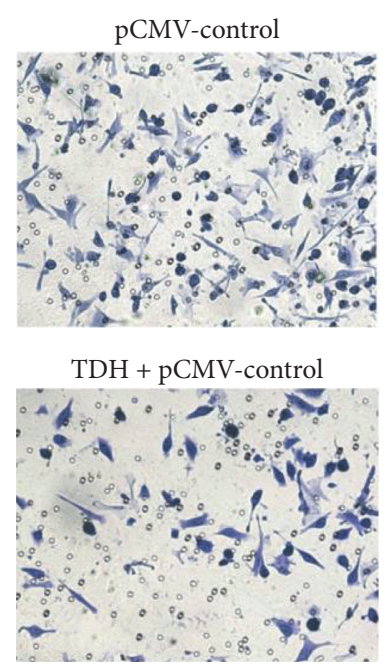

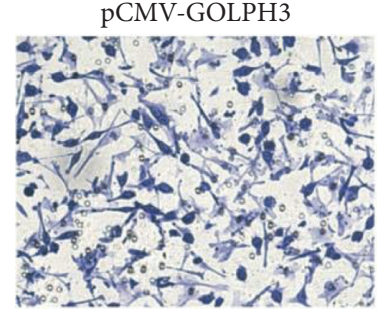

TDH + pCMV-GOLPH3

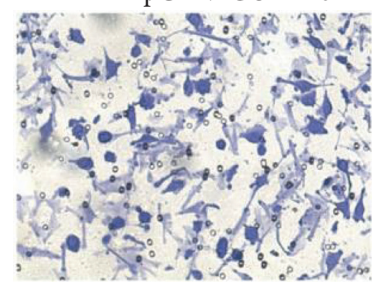

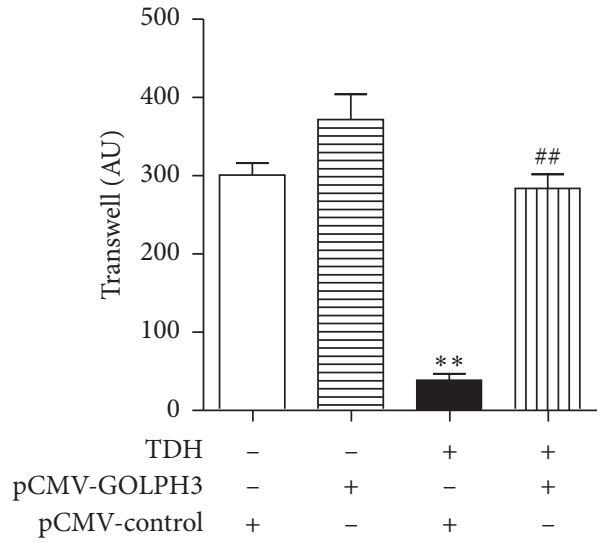

pCMV-control

FIgURE 7: Effect of TDH on migration in LoVo cells overexpressed GOLPH3 under TDH treatment. Experimental Group 3 compared with the p-CMV-2 Group ( $\left.{ }^{* *} P<0.01\right)$; Experimental Group 4 compared with Experimental Group $3\left({ }^{\# \#} P<0.01\right)$.

malignant tumor tissues, including glioma and liver, lung, ovarian, and esophageal cancer, and is positively correlated with poor prognosis [21-25]. It is also reported that GOLPH3 overexpression can activate various signaling pathways, promote cell proliferation, inhibit apoptosis, and induce the sensitivity of colon cancer cells to cisplatin and 5-FU $[7,8]$. In the present study, under the same condition of TDH treatment, compared with nontransfected LoVo cells, p-p70S6K and $\beta$-catenin expression and cell migration increased in LoVo cells overexpressed GOLPH3; these results suggested that the antitumor effect of TDH on LoVo cells was related to inhibition of the $\mathrm{PI} 3 \mathrm{~K} / \mathrm{AKT} / \mathrm{mTOR}$ and $\mathrm{Wnt} / \beta$-catenin signaling pathways through downregulating GOLPH3 gene expression. Therefore, we can reasonably speculate that $\mathrm{TDH}$ can block the resistance of colon cancer cells to cisplatin and 5-FU and improve chemotherapeutic efficacy in patients with advanced colon cancer.

\section{Conclusion}

$\mathrm{TDH}$ can inhibit the proliferation and migration and induce apoptosis in human colon cancer LoVo cells. The mechanism is that TDH can inhibit the PI3K/AKT/mTOR and $\mathrm{Wnt} / \beta$-catenin signaling pathways by downregulating GOLPH3 gene expression.

\section{Data Availability}

The data used to support the findings of this study are available from the corresponding author upon request.

\section{Conflicts of Interest}

The authors declare no conflicts of interest.

\section{Acknowledgments}

The authors sincerely thank Yanta Guo from the Second Affiliated Hospital of Fujian Medical University (Quanzhou, Fujian, China), Mingzhen Wang from Xiamen Hospital of TCM (Xiamen, Fujian, China), and Zhixiong Chen from the
Fifth Hospital of Xiamen (Xiamen, Fujian, China) for providing experimental suggestions. This research was supported by the Traditional Chinese Medicine Research Project of Fujian Province (Grant no. 2017FJZYLC403), the Medical Innovation Project of Fujian Province (Grant no. 2017-CXB-7), the Science and Technology Project of Quanzhou (Grant no. 2017Z015), and the Educational Research Project for Young and Middle-Aged Teachers of Fujian Educational Committee (Grant no. JAT190216).

\section{References}

[1] F. Bray, J. Ferlay, I. Soerjomataram, R. L. Siegel, L. A. Torre, and A. Jemal, "Global cancer statistics 2018: GLOBOCAN estimates of incidence and mortality worldwide for 36 cancers in 185 countries," CA: A Cancer Journal for Clinicians, vol. 68, no. 6, pp. 394-424, 2018.

[2] Z. Yan, Z. Lai, and J. Lin, "Anticancer properties of traditional Chinese medicine," Comb Chem High Throughput Screen, vol. 20, no. 5, pp. 423-429, 2017.

[3] X. Wang, Y. Yan, X. Chen et al., "The antitumor activities of Marsdenia tenacissima," Front Oncology, vol. 8, p. 473, 2018.

[4] C. Zhao, L.-Y. Han, W. Ren et al., "Metabolic profiling of tenacigenin $\mathrm{B}$, tenacissoside $\mathrm{H}$ and tenacissoside I using UHPLC-ESI-Orbitrap MS/MS," Biomedical Chromatography, vol. 30, no. 11, pp. 1757-1765, 2016.

[5] Y.-T. Guo, C. Z. Qiu, Z. X. Huang, W. S. Yu, X. F. Yang, and M. Z. Wang, "Correlational research of Golgi phosphorylation protein 3 expression in colorectal cancer," World Journal of Gastroenterology, vol. 21, no. 48, pp. 13473-13479, 2015.

[6] C.-Z. Qiu, M.-Z. Wang, W.-S. Yu, Y.-T. Guo, C.-X. Wang, and X.-F. Yang, "Correlation of GOLPH3 gene with Wnt signaling pathway in human colon cancer cells," Journal of Cancer, vol. 7, no. 8, pp. 928-934, 2016.

[7] M. Z. Wang, C. Z. Qiu, W. S. Yu, Y. T. Guo, C. X. Wang, and Z. X. Chen, "GOLPH3 expression promotes the resistance of HT29 cells to 5-fluorouracil by activating multiple signaling pathways," Molecular Medicine Reports, vol. 17, no. 1, pp. 542-548, 2018.

[8] Z. P. Zhou, L. P. Wang, Z. S. Hong et al., "Silencing GOLPH3 gene expression reverses resistance to cisplatin in HT29 colon cancer cells via multiple signaling pathways," International Journal of Oncology, vol. 53, no. 3, pp. 1183-1192, 2018. 
[9] Hospital authority of national Health and family planning commission of the People's Republic of China and Chinese society of oncology., "Chinese protocol of diagnosis and treatment of colorectal cancer," Zhonghua Wai Ke Za Zhi, vol. 56, no. 4, pp. 241-258, 2018.

[10] L. Yan and L. Ma, "Bulibu jillishan and kebinu mijiti, "application of traditional Chinese medicine in the adjuvant treatment of colon cancer," Chinese Pharmacist, vol. 21, no. 9, pp. 133-136, 2018.

[11] F. Yu, Y. Li, J. Zou et al., "The Chinese herb Xiaoaiping protects against breast cancer chemotherapy-induced alopecia and other side effects: a randomized controlled trial," Journal of International Medical Research, vol. 47, no. 6, pp. 2607-2614, 2019.

[12] A.-W. Zheng, Y.-Q. Chen, J. Fang, Y.-L. Zhang, and D.-D. Jia, "Xiaoaiping combined with cisplatin can inhibit proliferation and invasion and induce cell cycle arrest and apoptosis in human ovarian cancer cell lines," Biomedicine \& Pharmacotherapy, vol. 89, pp. 1172-1177, 2017.

[13] F. Wang, Q. X. Fan, H. H. Wang, D. M. Han, N. S. Song, and H. Lu, "Efficacy and safety of Xiaoaiping combined with chemotherapy in the treatment of advanced esophageal cancer," Zhonghua Zhong Liu Za Zhi, vol. 39, no. 6, pp. 453-457, 2017.

[14] Y. S. Jia, X. Q. Hu, H. Gabriella, L. J. Qin, and N. Meggyeshazi, "Antitumor activity of tenacissoside $\mathrm{H}$ on esophageal cancer through arresting cell cycle and regulating PI3K/Akt-NF- $\kappa \mathrm{B}$ transduction cascade," Evid Based Complement Alternative Medicine, vol. 2015, Article ID 464937, 2015.

[15] W. J. Wei, G. Q. Zhang, S. J. Liu, and T. X. Liu, “Anti-tumor effect of tenacissoside $\mathrm{H}$ and influence on immune function of lewis lung cancer mice," Chinese Journal of Traditional Chinese Medicine, vol. 21, no. 4, pp. 63-66, 2014.

[16] G. Hou, Q. Zhao, M. Zhang et al., "LSD1 regulates Notch and $\mathrm{PI} 3 \mathrm{~K} / \mathrm{Akt} / \mathrm{mTOR}$ pathways through binding the promoter regions of Notch target genes in esophageal squamous cell carcinoma," OncoTargets and Therapy, vol. 12, pp. 5215-5225, 2019.

[17] J. Deng, X. Bai, X. Feng et al., "Inhibition of PI3K/Akt/mTOR signaling pathway alleviates ovarian cancer chemoresistance through reversing epithelial-mesenchymal transition and decreasing cancer stem cell marker expression," BMC Cancer, vol. 19, no. 1, p. 618, 2019.

[18] P.-W. Lu, L. Li, F. Wang, and Y.-T. Gu, "Inhibitory role of large intergenic noncoding RNA-ROR on tamoxifen resistance in the endocrine therapy of breast cancer by regulating the PI3K/Akt/mTOR signaling pathway," Journal of Cellular Physiology, vol. 234, no. 2, pp. 1904-1912, 2019.

[19] X. Tan, Z. Zhang, H. Yao, and L. Shen, "Tim-4 promotes the growth of colorectal cancer by activating angiogenesis and recruiting tumor-associated macrophages via the PI3K/AKT/ mTOR signaling pathway," Cancer Letters, vol. 436, pp. 119-128, 2018.

[20] X. Cheng, X. Xu, D. Chen, F. Zhao, and W. Wang, "Therapeutic potential of targeting the $\mathrm{Wnt} / \beta$-catenin signaling pathway in colorectal cancer," Biomedicine \& Pharmacotherapy, vol. 110, pp. 473-481, 2019.

[21] X. Zhou, S. Xie, S. Wu et al., "Golgi phosphoprotein 3 promotes glioma progression via inhibiting Rab5-mediated endocytosis and degradation of epidermal growth factor receptor," Neuro-Oncology, vol. 19, no. 12, pp. 1628-1639, 2017.

[22] H. Liu, X. Wang, B. Feng et al., "Golgi phosphoprotein 3 (GOLPH3) promotes hepatocellular carcinoma progression by activating mTOR signaling pathway," BMC Cancer, vol. 18, no. 1, p. 661, 2018.

[23] S. Tang, R. Yang, X. Zhou, H. Pan, and J. Liu, "Expression of GOLPH3 in patients with non-small cell lung cancer and xenografts models," Oncology Letters, vol. 15, no. 15, pp. 7555-7562, 2018.

[24] J. Sun, X. Yang, R. Zhang et al., "GOLPH3 induces epithelialmesenchymal transition via $\mathrm{Wnt} / \beta$-catenin signaling pathway in epithelial ovarian cancer," Cancer Medicine, vol. 6, no. 4, pp. 834-844, 2017.

[25] J.-H. Wang, L.-J. Yuan, R.-X. Liang et al., "GOLPH3 promotes cell proliferation and tumorigenicity in esophageal squamous cell carcinoma via mTOR and Wnt/ $\beta$-catenin signal activation," Molecular Medicine Reports, vol. 16, no. 5, pp. 71387144, 2017. 